\title{
Clinical and MRI evaluation of anxiety as the first symptom of sporadic Creutzfeldt-Jakob disease: A case report
}

\author{
WEIBO LIU ${ }^{1}$, YUNRONG LU ${ }^{1}$, GUODONG ZHONG ${ }^{2}$, BIAO JIANG ${ }^{3}$ and ZHIJIE PAN ${ }^{4}$ \\ Departments of ${ }^{1}$ Psychiatry, ${ }^{2}$ Neurology and ${ }^{3}$ Radiology, The Second Affiliated Hospital; \\ ${ }^{4}$ Department of Respiratory Medicine, The First Affiliated Hospital, School of Medicine, \\ Zhejiang University, Hangzhou, Zhejiang 310003, P.R. China
}

Received November 19, 2014; Accepted December 22, 2015

DOI: $10.3892 / \mathrm{etm} .2016 .3041$

\begin{abstract}
Sporadic Creutzfeldt-Jakob disease (sCJD) is a rare, transmissible prion disease of the brain, characterized by prominent neurological symptoms and progressive dementia. Early psychiatric manifestations as an initial or sole symptom of sCJD are relatively rare. The current report describes an elderly female patient with SCJD demonstrating anxiety as an initial symptom. The present patient was initially diagnosed with adjustment disorder and anxiety; however, the rapid deterioration of the patient's cognitive and neurological functioning led to a diagnosis of sCJD. Diffusion-weighted magnetic resonance images of the brain supported a diagnosis of SCJD, and it was posited that the abnormalities of gray matter that were observed within the bilateral cingulate cortex may be the pathophysiological basis of the anxiety associated with this case of SCJD. The patient eventually succumbed to inhalational bronchopneumonia 5 months after anxiety onset. The present case report emphasizes the importance of secondary causes of anxiety symptoms in elderly patients, and indicates that brain DWI is a non-invasive and useful examination in the early diagnosis of sCJD.
\end{abstract}

\section{Introduction}

Sporadic Creutzfeldt-Jakob disease (sCJD) is a rare, transmissible and ultimately fatal prion disease of the brain, characterized by rapidly progressing dementia. This is often accompanied by myoclonus, multiple symptoms of neurological dysfunction and a periodic electroencephalogram pattern $(1,2)$. sCJD is typically characterized by prominent neurological symptoms, including myoclonus, visual or cerebellar disturbance, pyramidal/extrapyramidal dysfunction and

Correspondence to: Dr Zhijie Pan, Department of Respiratory Medicine, The First Affiliated Hospital, School of Medicine, Zhejiang University,79 Qingchun Road,Hangzhou,Zhejiang 310003,P.R.China E-mail: drzjpan@163.com

Key words: diffusion-weighted images, sporadic Creutzfeldt-Jakob disease, anxiety, dementia, cingulate cortex akinetic mutism (3), but less commonly with early psychiatric manifestation. There are a number of reports suggesting that psychiatric manifestations may occur as a symptom during the development of sCJD $(2,4)$, but early psychiatric manifestations as an initial or sole symptom of SCJD are relatively rare. The present study describes a 67-year-old woman who first presented with anxiety symptoms but, following initial diagnosis with adjustment disorder and anxiety, was subsequently diagnosed with probable sCJD. Diffusion-weighted images (DWIs) supported this diagnosis of sCJD.

\section{Case report}

A 67-year-old woman was admitted to the inpatient psychiatric service of the Second Affiliated Hospital of Zhejiang University School of Medicine (Hangzhou, China) with anxiety and insomnia, which had begun a month prior to admission, and had been initiated by family issues. The patient was admitted to the neurology clinic 2 weeks later due to dizziness, but the neurological examination results and brain magnetic resonance imaging (MRI) T1-weighted images (T1WIs) and T2-weighted images (T2WIs) were normal [captured using a Sonata 1.5T MRI scanner (Siemens AG, Munich, Germany)]. The patient reported that the symptoms of anxiety subsequently increased, and that she became increasingly dependent on others, but remained able to perform normal activities. The patient had experienced similar anxiety symptoms a year previously, following a bereavement; however, the patient fully recovered after 1 month of treatment with the anxiolytic drugs citalopram (20 mg, daily) and lorazepam ( $0.5 \mathrm{mg}$, at bedtime). The patient had no history of alcohol or drug abuse, memory problems, surgery or any other noteworthy prior medical history. Furthermore, there was no reported family history of any neuropsychiatric diseases.

An initial neurological examination revealed a bilateral mild fluctuating tremor in the hands, which was not apparent when the patient was relaxed or distracted. Upon examination of mental status, the patient appeared anxious and restless, but exhibited no disorientation, hallucinations or delusions. Psychological testing using the Hamilton Anxiety Rating Scale (HAM-A) revealed a score of 23, indicative of moderate anxiety (5). Furthermore, the patient exhibited no clear cognitive impairment; the patient recorded a Mini Mental Status 


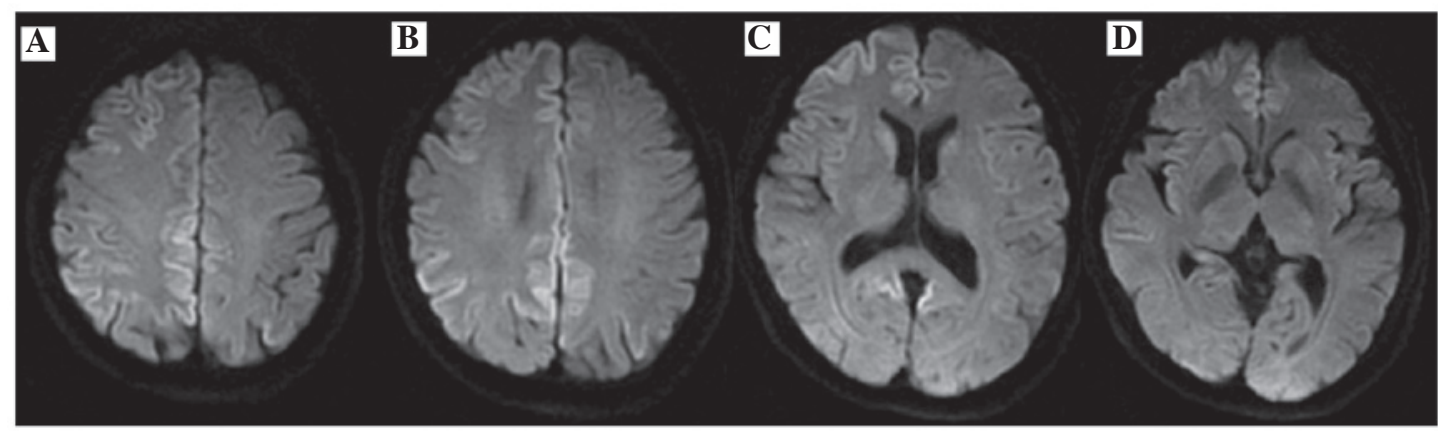

Figure 1. Axial brain magnetic resonance images. High signal intensity in diffusion-weighted images in the (A) right superior prefrontal cortex, right precentral gyrus and bilateral superior parietal lobes, (B) bilateral cingulate cortex, (C) head of the caudate nucleus and (D) insula, revealing abnormally high, ribbon-like cortical signalling. (B-D) Hyperintense signals appearing symmetrically in the bilateral cingulate cortex are particularly pronounced.

Examination (MMSE) score of 26/30 and a Wechsler Adult Intelligence Scale-Revised by China (WAIS-RC) score of 90 (6,7). Initial electroencephalogram (EEG; sourced from Shanghai Nuocheng Electric Appliance Co., Ltd., Shanghai, China) analysis demonstrated mildly abnormal brain activity, with slowed alpha rhythms.

The initial diagnosis was of adjustment disorder and anxiety, and the patient was prescribed citalopram (20 mg, daily) and alprazolam ( $0.4 \mathrm{mg}$, at bedtime) to relieve anxiety. Upon assessment 10 days later, the psychiatric symptoms had improved and the HAM-A score had reduced to 11; however, the patient had become increasingly forgetful and demonstrated slowed performance in daily functioning. Cognitive appraisal revealed a deterioration in cognition, with an MMSE score of 18/30 and a WMS-CR score of 55. The patient had a staggering gait and occasional choreic movement of the upper extremities. An EEG revealed moderately abnormal brain activity, with enhanced theta activity. A lumbar puncture was performed; an analysis of cerebrospinal fluid (CSF) demonstrated no marked alterations. However, examination of 14-3-3 protein and tau protein was not possible in the current hospital. When examined by MRI, no abnormality was detected on the brain T1WI and T2WI sequences, but DWI revealed abnormally high, ribbon-like cortical signal intensity within numerous brain regions, particularly within the bilateral cingulate cortex (Fig. 1). Following consultation with a neurologist, the patient was diagnosed with possible sCJD and transferred as an inpatient to the neurology department. The patient was monitored and treated with acyclovir $(1,500 \mathrm{mg}$, daily) and citicoline (500 mg, daily) but developed increasing confusion, dysphasia, tremors, myoclonus and ataxia. A neurological examination revealed cogwheel rigidity, bilaterally increased deep tendon reflexes and positive Babinski reflexes. A severe deterioration of the patient's cognition was observed, with an MMSE score of 4/30. Serial EEG demonstrated periodic sharp-wave complexes. In accordance with the World Health Organization criteria (3), the patient was diagnosed with probable sCJD 2 weeks after diagnosis with possible sCJD. Following this, the patient deteriorated rapidly, experiencing progressive confusion, ataxia, myoclonus and frequent convulsions. The patient became bedbound and succumbed to inhalational bronchopneumonia 5 months after the onset of anxiety. A postmortem was not performed upon the request of relatives.

\section{Discussion}

In the current report, anxiety was presented as the sole initial symptom of sCJD in an elderly woman with a history of anxiety disorder, which was misdiagnosed as the primary psychiatric disorder and delayed the diagnosis of SCJD. The initial presentation of SCJD is quite variable, and the two most recognized cardinal features of SCJD are rapidly progressing dementia and myoclonus $(8,9)$. A previous cohort study of 114 patients with SCJD revealed that $20 \%$ of patients may present with 'behavioral symptoms', typically agitation, irritability and depression (10). However, reports of early psychiatric manifestations as an initial or sole symptom of SCJD are relatively rare. Wall et al (2) previously reported upon 2 patients with SCJD who were concerned regarding their health prior to the development of severe dementia. Jardri el al (11) reported a SCJD case demonstrating early depressive disorder with psychotic symptoms, which delayed the diagnosis of sCJD (11). Based on the incidence of psychiatric symptoms in the present and previous reports, it can be concluded that a number of patients may appear to have psychiatric symptoms prior to diagnosis of sCJD, and therefore are initially treated for psychiatric illness.

In the present case, the patient expressed anxiety due to an identifiable stressor during the prodromal phase of sCJD, and this anxiety persisted during the onset phase and cognitive deterioration. This anxiety prompted the patient to seek medical attention, and anxiolytic drugs were effective in its treatment. These symptoms were initially hypothesized to have psychogenic origin due to the reported history of anxiety and the absence of additional neurological symptoms. However, the progressive deterioration of cognitive and neurological symptoms raised suspicions of the presence of sCJD. Stressful life events have been established to be risk factors associated with several neurological and psychiatric diseases, and it is possible that brain lesions may act together with stressful events to lead to the manifestation of these diseases (12). In the present case, this suggests that increased anxiety, triggered by a stressful experience, may act together with brain legions to present as anxiety symptoms in patients with SCJD.

Thorough neurological, cognitive and psychiatric examinations are important for observation of the clinical features of sCJD. However, a definitive diagnosis of SCJD is reliant upon the neuropathological results of a brain biopsy, which presents substantial risks to the patient; furthermore, there is 
also an added risk of false-negative results associated with sampling error (13). Available tests include EEG examinations and the detection of 14-3-3 protein in the CSF, but neither test is specific or able to provide early detection of $\operatorname{sCJD}(9,14)$. As $\mathrm{SCJD}$ results from prion proliferation within the central nervous system, with cortical and subcortical manifestations, the guidelines from the University of California, San Francisco have advocated the use of MRI in the diagnosis of SCJD (15). Use of brain MRI provides a radiological method of sCJD diagnosis, and supports amendment of the current clinical diagnostic criteria for SCJD; use of DWI additionally increases the diagnostic sensitivity of MRI in the detection of sCJD (16,17). Bahn and Parchi (18) reported on 4 patients with SCJD demonstrating isolated to diffuse cortical ribbons using DWI, suggesting that DWI was a more sensitive method than T2WI for detecting gray matter abnormalities in SCJD sequences. Yaguchi et al (19) have previously suggested that standardizing DWI is useful in the early diagnosis of CJD. A multi-center international study aiming to improve the clinical diagnostic criteria for SCJD proposed that high signal abnormalities in the caudate nucleus and putamen or $>2$ cortical regions (in the parietal-temporal-occipital regions) using DWI may support a diagnosis of SCJD, and that the sensitivity of the criteria for the SCJD spectrum would improve from $92 \%$ (WHO criteria) to $98 \%$ (MRI-CJD Consortium) (17). In the current case, compared with the normal T1WI and T2WI sequences, the hyperintensities detected within the basal ganglia and cortical regions using DWI temporally preceded changes revealed using EEG to support the diagnosis of sCJD.

Evidence from brain imaging indicates that specific brain structures, such as the amygdaloid complex and the cingulate and prefrontal cortices, are mediators of the broad range of behavioral and physiological responses associated with anxiety (20). The examination of patients suffering from anxiety using functional MRI has demonstrated increased activity in the inferior frontal cortex, hippocampus and throughout the anterior and posterior cingulate cortices, which provides support for the hypothesis that the limbic circuit is involved in the pathophysiology of anxiety (21). In the present study, DWI revealed remarkably restricted diffusion amongst multiple brain regions, particularly in the bilateral cingulate cortices. As the cingulate cortex is a section of the limbic lobe involved with generation of emotions, it is possible that the gray matter abnormality of bilateral cingulate cortex may be associated with the etiology of anxiety symptoms.

In conclusion, the present case demonstrates that $\mathrm{SCJD}$ may affect moods in its early stages, and present anxiety as the first symptom. The importance of secondary causes of anxiety symptoms in elderly patients should be noted, however, and a global approach and differential diagnosis are additionally required in the evaluation of primarily psychiatric syndromes. Brain DWI is a non-invasive and useful adjuvant examination for the early diagnosis of sCJD, which may present with initial psychiatric symptoms.

\section{Acknowledgements}

The present study was supported by a grant from Zhejiang Science and Technology Research Fund in China (grant no. 2012C33119) and Zhejiang Traditional Medical Science Research Fund in China (grant no. 2010ZB059).

\section{References}

1. Brown P, Gibbs CJ Jr, Rodgers-Johnson P, Asher DM, Sulima MP, Bacote A, Goldfarb LG and Gajdusek DC: Human spongiform encephalopathy: The National Institutes of Health series of 300 cases of experimentally transmitted disease. Ann Neurol 35: 513-529, 1994

2. Wall CA, Rummans TA, Aksamit AJ, Krahn LE and Pankratz VS: Psychiatric manifestations of Creutzfeldt-Jakob disease: A 25-year analysis. J Neuropsychiatry Clin Neurosci 17: 489-495, 2005.

3. World Health Organization (WHO): Global surveillance, diagnosis and therapy of human Transmissible Spongiform Encephalopathies: Report of a WHO consultation. Geneva, Switzerland, 9-11 February 1998. Available from: apps.who. int/iris/bitstream/10665/65516/1/WHO EMC ZDI 98.9.pdf.

4. Chuang DT, O'Dowd MA, Frieder A, Haut SR and Robbins MS: Delayed diagnosis of sporadic Creutzfeldt-Jakob disease in a patient with schizophrenia. Psychosomatics 53: 392-396, 2012.

5. Hamilton $\mathrm{M}$ : The assessment of anxiety states by rating. $\mathrm{Br} \mathrm{J}$ Med Psychol 32: 50-55, 1959.

6. Rovner BW and Folstein MF: Mini-mental state exam in clinical practice. Hosp Pract (Off Ed) 22: 99, 103, 106, 110, 1987.

7. Gong YX: Revision of Wechsler Adult Intelligence Scale in China. Acta Psychologica Sinica 15: 362-369, 1983.

8. Appleby BS, Appleby KK and Rabins PV: Does the presentation of Creutzfeldt-Jakob disease vary by age or presumed etiology? A meta-analysis of the past 10 years. J Neuropsychiatry Clin Neurosci 19: 428-435, 2007.

9. Moellentine CK and Rummans TA: The varied neuropsychiatric presentations of Creutzfeldt-Jakob disease. Psychosomatics 40: 260-263, 1999.

10. Rabinovici GD, Wang PN, Levin J, Cook L, Pravdin M, Davis J, DeArmond SJ, Barbaro NM, Martindale J, Miller BL and Geschwind MD: First symptom in sporadic Creutzfeldt-Jakob disease. Neurology 66: 286-287, 2006.

11. Jardri R, DiPaola C, Lajugie C, Thomas P and Goeb JL: Depressive disorder with psychotic symptoms as psychiatric presentation of sporadic Creutzfeldt-Jakob disease: A case report. Gen Hosp Psychiatry 28: 452-454, 2006.

12. Esch T, Stefano GB, Fricchione GL and Benson H: The role of stress in neurodegenerative diseases and mental disorders. Neuro Endocrinol Lett 23: 199-208, 2002.

13. Tartaro A, Fulgente T, Delli Pizzi C, Bonomo L, Bocola V and Onofrj M: MRI alterations as an early finding in Creutzfeld-Jakob disease. Eur J Radiol 17: 155-158, 1993.

14. Collins SJ, Sanchez-Juan P, Masters CL, Klug GM, van Duijn C, Poleggi A, Pocchiari M, Almonti S, Cuadrado-Corrales N, de Pedro-Cuesta J, et al: Determinants of diagnostic investigation sensitivities across the clinical spectrum of sporadic Creutzfeldt-Jakob disease. Brain 129: 2278-2287, 2006.

15. Geschwind MD: Rapidly progressive dementia: Prion diseases and other rapid dementias. Continuum (Minneap Minn) 16: 31-56, 2010.

16. Young GS, Geschwind MD, Fischbein NJ, Martindale JL, Henry RG, Liu S, Lu Y, Wong S, Liu H, Miller BL and Dillon WP: Diffusion-weighted and fluid-attenuated inversion recovery imaging in Creutzfeldt-Jakob disease: High sensitivity and specificity for diagnosis. AJNR Am J Neuroradiol 26: 1551-1562, 2005

17. Zerr I, Kallenberg K, Summers DM, Romero C, Taratuto A, Heinemann U, Breithaupt M, Varges D, Meissner B, Ladogana A et al: Updated clinical diagnostic criteria for sporadic Creutzfeldt-Jakob disease. Brain 132: 2659-2668, 2009.

18. Bahn MM and Parchi P: Abnormal diffusion-weighted magnetic resonance images in Creutzfeldt-Jakob disease. Arch Neurol 56: 577-583, 1999.

19. Yaguchi M, Yaguchi $\mathrm{H}$ and Kanaya T: Early lesion of Creutzfeldt-Jakob disease detected by standardizing diffusion-weighted images. Intern Med 48: 1717-1718, 2009.

20. Davidson RJ: Anxiety and affective style: Role of prefrontal cortex and amygdala. Biol Psychiatry 51: 68-80, 2002.

21. Bystritsky A, Pontillo D, Powers M, Sabb FW, Craske MG and Bookheimer SY: Functional MRI changes during panic anticipation and imagery exposure. Neuroreport 12: 3953-3957, 2001. 